\title{
Dynamic stress intensity factors of interacting branched cracks
}

\author{
Piotr Fedeliński ${ }^{1, *}$ \\ ${ }^{1}$ Silesian University of Technology, 44-100 Gliwice, Poland
}

\begin{abstract}
In the present work the boundary element method (BEM) is applied to analysis of statically and dynamically loaded infinite plates with multiple stationary branched cracks. The material of the plates is linear-elastic, homogenous and isotropic. In the applied BEM approach the displacement and traction boundary integral equations are used simultaneously for nodes on crack surfaces. Contrary to the finite element method (FEM) in the BEM numerical solutions are obtained by discretization of external boundaries and crack surfaces. The dynamic problem is solved by using the Laplace transform method and the solution in the time domain is computed by the Durbin numerical inversion method. Numerical examples of multiple branched cracks in infinite plates subjected to static and dynamic loadings are presented. An influence of orientation, distances between cracks and the number of cracks on static and dynamic stress intensity factors (SIF) is studied.
\end{abstract}

\section{Introduction}

Branched cracks may be material defects that arise during the manufacture of materials or structures and as a result of exploitation. Single cracks can branch out during propagation, especially under dynamic loading. If there are many cracks in the material then they interact with each other causing a change in the stress fields. This interaction depends on the orientation, distance and number of cracks. The purpose of the work is to determine the influence of crack interaction on static and dynamic stress intensity factors (SIF), which characterize stresses near the tips of cracks.

Daux et al. [1] presented the extended finite element method (X-FEM) to analysis of cracks with multiple branches and cracks emanating from holes. A standard displacement approximation was enriched by incorporating additional discontinuous functions. The method allows the modelling of discontinuities independently of the mesh. Raffie et al. [2] investigated bifurcation and trifurcation of fast running cracks under various biaxial loading conditions. Arbitrary curvilinear crack propagations were analyzed by the time-domain BEM. Branching of cracks was controlled by the opening mode SIF and velocity and direction of crack growth by the maximum circumferencial stress at the crack tip. Numerical solutions were compared with experimental results.

* Corresponding author: piotr.fedelinski@polsl.pl 
In the present work the boundary element method (BEM) is applied to analysis of statically and dynamically loaded branched cracks in infinite plates. The crack problem is solved by the dual BEM which was developed for static loadings by Portela et al. [3] and for dynamical loadings by Fedeliński et al. [4]. An overview of different BEM approaches in dynamic fracture mechanics was presented by Fedeliński [5]. The application of the method for single branched cracks was shown by Fedeliński in [6]. In this work the Laplace transform method is applied to analysis of interacting branched cracks in infinite plates.

In the dual BEM only boundaries of the body and crack surfaces are divided into boundary elements. The variations of boundary coordinates, displacements and tractions are interpolated using shape functions and nodal values. The relations between boundary displacements and tractions are expressed by the displacement and traction boundary integral equations. The displacement equation is applied for nodes on the external boundary and both equations for nodes on crack surfaces. The time dependent problems are solved using the Laplace transform method and the solution in the time domain is computed by the Durbin numerical inversion method. In the dual BEM the boundary displacements and tractions are computed directly. Static stress intensity factors (SIF) are calculated using the path independent J-integral and the dynamic SIF using crack opening displacements.

\section{Numerical examples - branched cracks in an infinite domain}

Static and dynamic stress intensity factors (SIF) of multiple branched cracks in an infinite domain are analyzed. The material of the plate has the following elastic properties: the Young modulus $E=200 \mathrm{GPa}$, the Poisson ratio $v=0.3$, the mass density $\rho=8000 \mathrm{~kg} / \mathrm{m}^{3}$ and the plate is in the plane strain conditions. The velocity of the longitudinal wave is $c_{1}=5801 \mathrm{~m} / \mathrm{s}$ and the velocity of the shear wave is $c_{2}=3101 \mathrm{~m} / \mathrm{s}$. The plate is loaded by the Heaviside time dependent uniformly distributed tension $p$ applied at the initial time $t=0$. The static tension has the constant value $p$. The numerical solution is obtained using 50 Laplace parameters and the time step is $\Delta t=0.5 \mu \mathrm{s}$. In order to model an infinite domain a large square plate having dimensions $h=w=15 \mathrm{~cm}$ is considered, as shown in Fig. 1a. The plate is constrained along the right edge and loaded along the left edge. The branched cracks are situated near the center of the plate $O$ (Fig. 1b). The cracks have three branches of length $a=1 \mathrm{~cm}$ and the angle between the branches is $2 / 3 \pi$. The distance of crack centers from the point $O$ is $l$, unless otherwise defined. The crack tips of the horizontal and inclined branches are denoted by $A$ and $B$, respectively. The length of the cracks in the vertical direction is $2 c_{2}$. The SIF are normalized with respect to the static SIF $K_{o}=p\left(\pi c_{2}\right)^{1 / 2}$ for the straight crack of length $2 c_{2}$ in an infinite domain loaded by the remote tension $p$. The external boundary of the square plate is divided into 200 boundary elements and each branched crack into 60 boundary elements having the same length.

a)

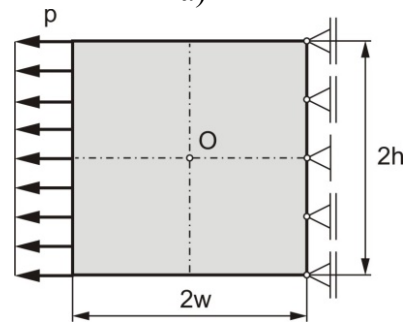

b)

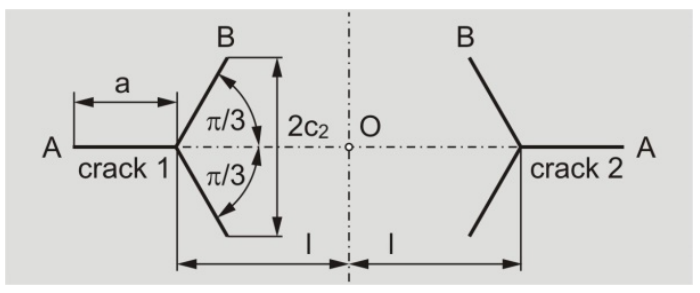

Fig. 1. Branched cracks in a square plate: a) dimensions of the plate and boundary conditions, b) dimensions of branched cracks. 


\subsection{Different orientations of two branched cracks in an infinite domain}

a)

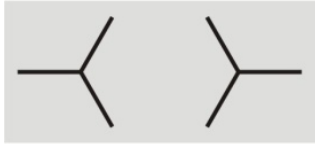

c)

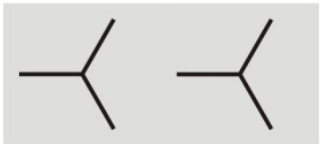

b)

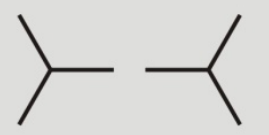

d)

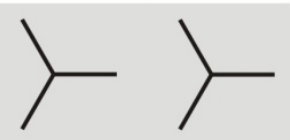

Fig. 2. Orientation of two branched cracks in an infinite domain.

Four different orientations of cracks shown in Fig. 2 are considered. The distance of crack centers from the point $O$ is $l=1.5 \mathrm{~cm}$. The normalized static SIF $K / K_{o}$ are given in Table 1. Because of the symmetry of the cracks the results are not shown for the crack 2 for the case (a) and (b) and for the case (d). The results are compared with the solutions for the single crack. The SIF $K_{I} / K_{o}(A)$ are very small and the second crack has small influence on the values. The SIF $K_{I} / K_{o}(B)$ has smaller values when the crack tips $B$ are in a small distance (case (a) and (c)). The SIF $K_{I I} / K_{o}(B)$ for the first crack is not sensitive to the orientation of cracks. The $\operatorname{SIF} K / K_{o}(B)$ for the inclined branch $B$ in each case are smaller than for the single crack.

Table 1. Normalized SIF for different orientations of two branched cracks in an infinite domain.

\begin{tabular}{|c|c|c|c|c|c|c|}
\hline \multirow{2}{*}{ orientation } & \multicolumn{3}{|c|}{ crack 1 } & \multicolumn{3}{c|}{ crack 2 } \\
\cline { 2 - 7 } & $\boldsymbol{K}_{\boldsymbol{I}}(\boldsymbol{A})$ & $\boldsymbol{K}_{\boldsymbol{I}}(\boldsymbol{B})$ & $\boldsymbol{K}_{\boldsymbol{I I}}(\boldsymbol{B})$ & $\boldsymbol{K}_{\boldsymbol{I}}(\boldsymbol{A})$ & $\boldsymbol{K}_{\boldsymbol{I}}(\boldsymbol{B})$ & $\boldsymbol{K}_{\boldsymbol{I I}}(\boldsymbol{B})$ \\
\hline $\mathrm{a}$ & 0.029 & 0.628 & 0.490 & - & - & - \\
\hline b & 0.051 & 0.727 & 0.489 & - & - & - \\
\hline c & 0.028 & 0.674 & 0.500 & 0.065 & 0.717 & 0.464 \\
\hline single & 0.016 & 0.754 & 0.541 & - & - & - \\
\hline
\end{tabular}

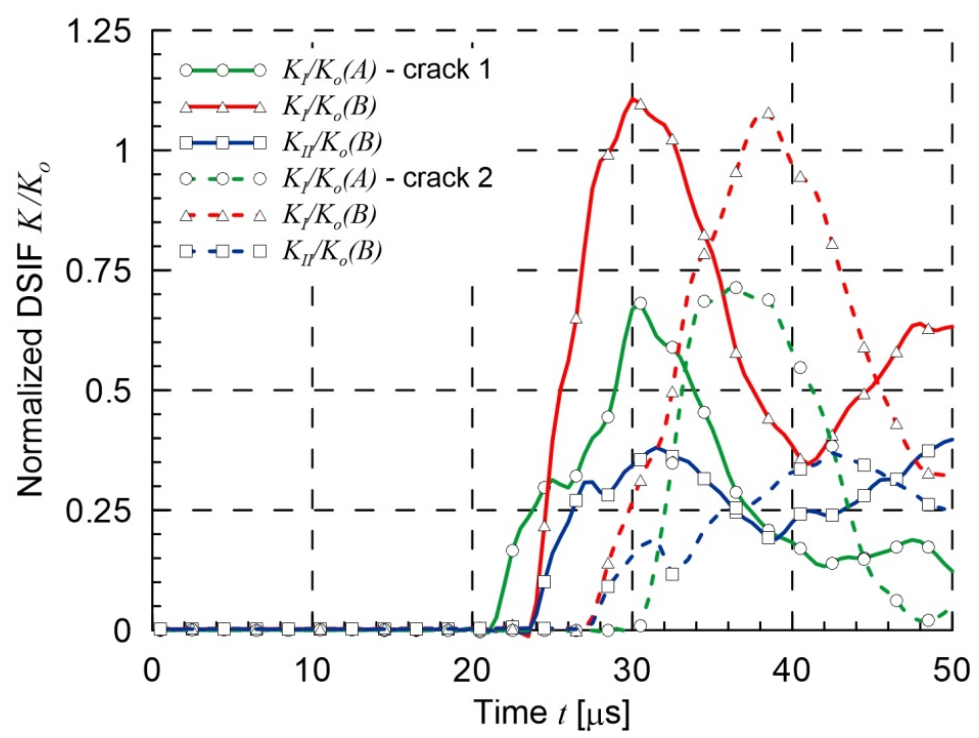

Fig. 3. Normalized DSIF $K / K_{o}$ for the orientation of cracks $(a)$. 


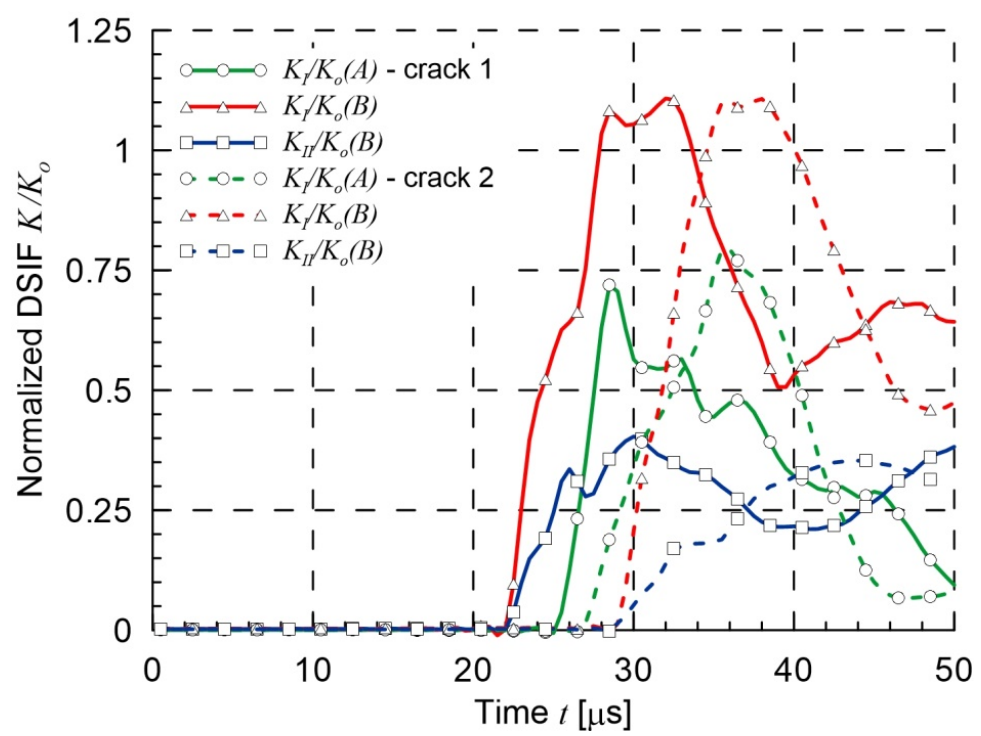

Fig. 4. Normalized DSIF $K / K_{o}$ for the orientation of cracks $(b)$.

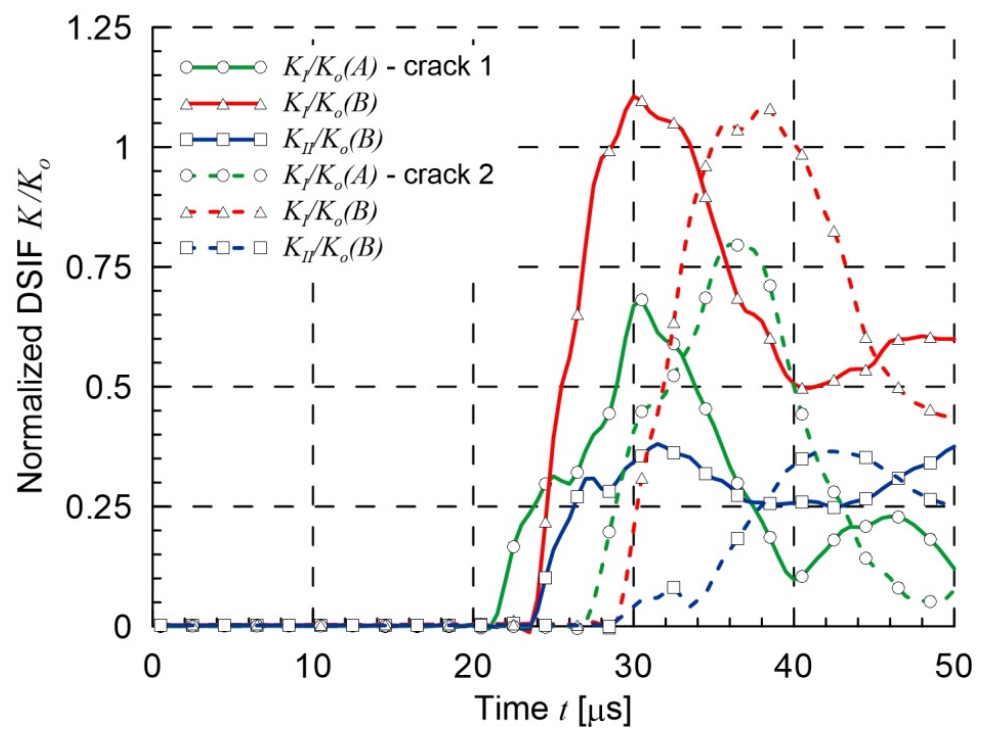

Fig. 5. Normalized DSIF $K / K_{o}$ for the orientation of cracks $(c)$.

The normalized dynamic DSIF $K / K_{o}$ for different orientations are presented in Fig. 3-6. The longitudinal wave propagates from the loaded boundary to the crack tip $A$ in cases (a) and (c) $21.5 \mu \mathrm{s}$ and to the crack tip $B$ in cases (b) and (d) $22.4 \mu \mathrm{s}$. During this time the cracks are at rest and the DSIF are zero. The time dependences of DSIF for each configuration of cracks are different but the maximum values are similar. The static SIF for the horizontal branches are small but the dynamic DSIF have significant values. The normalized dynamic DSIF $K_{I} / K_{o}(B)$ are larger than $K_{I} / K_{o}(A)$. 


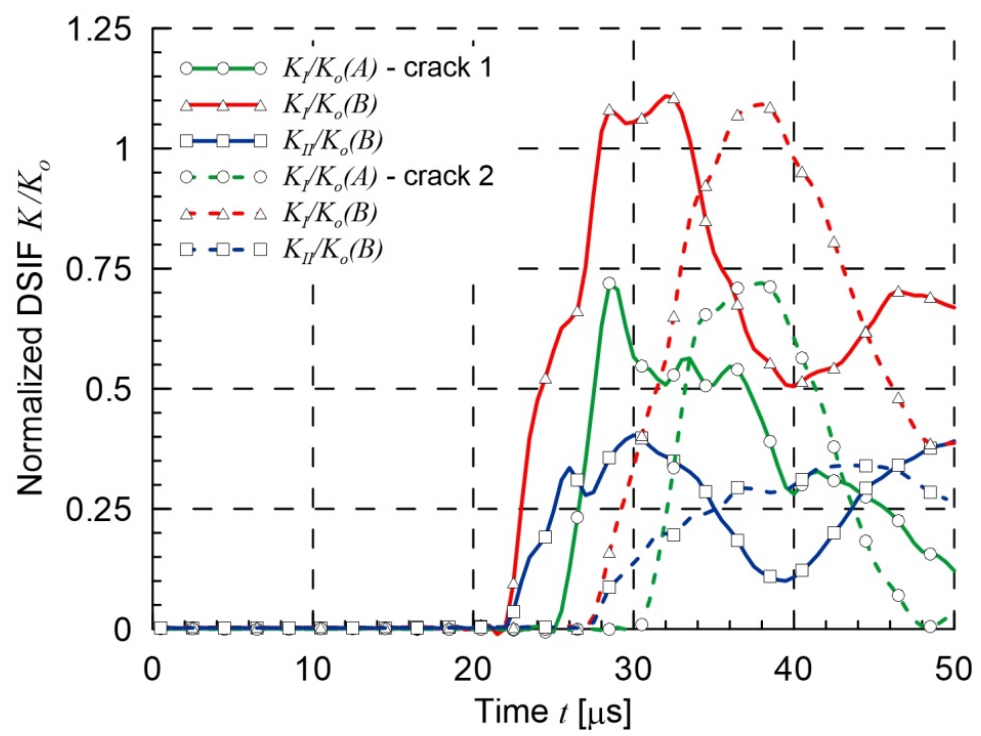

Fig. 6. Normalized DSIF $K / K_{o}$ for the orientation of cracks $(d)$.

\subsection{Different distance between two branched cracks in an infinite domain}

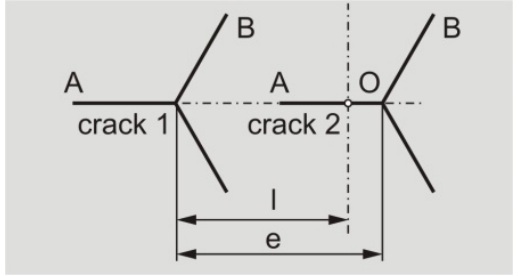

Fig. 7. Different distances between two branched cracks in an infinite domain.

The influence of the distance $e$ between crack centers on SIF for the configuration shown in Fig. 7 is studied. The distances are $e=2.0,2.5,3.0,3.5 \mathrm{~cm}$. The normalized static SIF $K / K_{o}$ are given in Table 2 . The results are compared with the solution for the single crack. When the distance increases the SIF for the inclined branches increase.

Table 2. Normalized SIF for different distances between two branched cracks in an infinite domain.

\begin{tabular}{|c|c|c|c|c|c|c|}
\hline distance & \multicolumn{3}{|c|}{ crack 1 } & \multicolumn{3}{c|}{ crack 2 } \\
\cline { 2 - 7 } $\boldsymbol{e} / \boldsymbol{l}$ & $\boldsymbol{K}_{\boldsymbol{I}}(\boldsymbol{A})$ & $\boldsymbol{K}_{\boldsymbol{I}}(\boldsymbol{B})$ & $\boldsymbol{K}_{\boldsymbol{I I}}(\boldsymbol{B})$ & $\boldsymbol{K}_{\boldsymbol{I}}(\boldsymbol{A})$ & $\boldsymbol{K}_{\boldsymbol{I}}(\boldsymbol{B})$ & $\boldsymbol{K}_{\boldsymbol{I I}}(\boldsymbol{B})$ \\
\hline $2.0 / 1.5$ & 0.032 & 0.614 & 0.488 & 0.014 & 0.700 & 0.416 \\
\hline $2.5 / 1.5$ & 0.031 & 0.649 & 0.493 & 0.068 & 0.709 & 0.441 \\
\hline $3.0 / 1.5$ & 0.028 & 0.674 & 0.500 & 0.065 & 0.717 & 0.464 \\
\hline $3.5 / 1.5$ & 0.027 & 0.692 & 0.506 & 0.055 & 0.722 & 0.480 \\
\hline single & 0.016 & 0.754 & 0.541 & - & - & - \\
\hline
\end{tabular}

The normalized dynamic DSIF $K_{I} / K_{o}$ for the crack tips $A$ and $B$ are shown in Fig. 8 and 9, respectively. The DSIF for the horizontal branch $A$ are larger for the second crack than for the first crack. The distance between the cracks have the significant influence on the $K_{I} / K_{o}(A)$ for the first crack for times larger than $36 \mu$ s (Fig. 8). The maximal values of DSIF $K_{I} / K_{o}(B)$ for the inclined branches are similar (Fig. 9). 


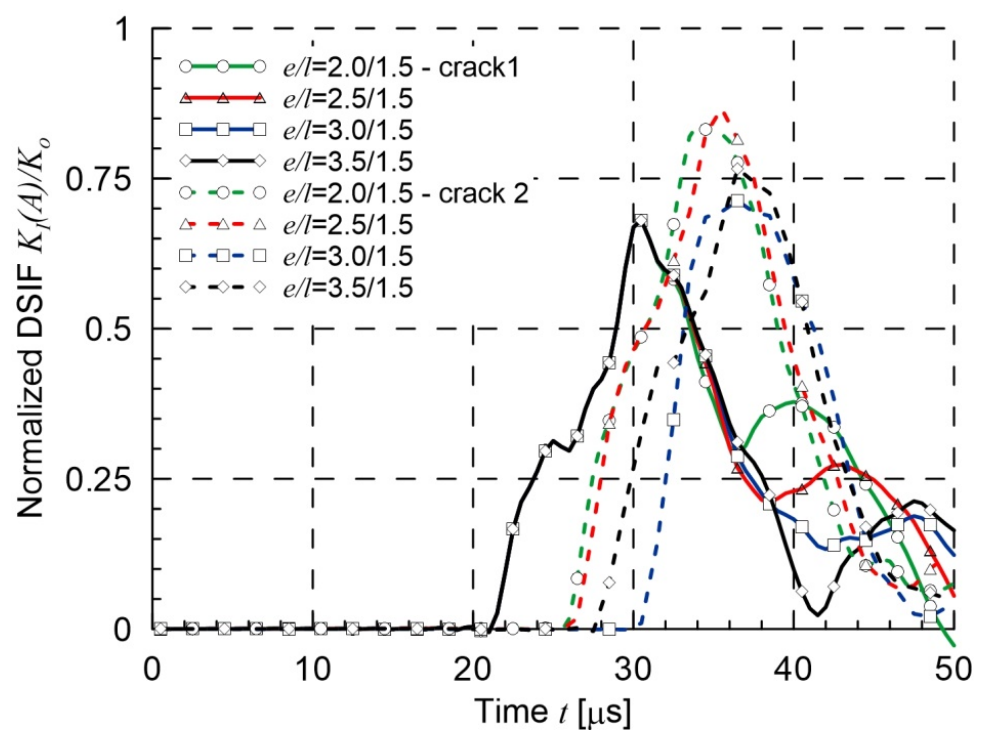

Fig. 8. Normalized DSIF $K_{I} / K_{o}(A)$ for the horizontal branches.

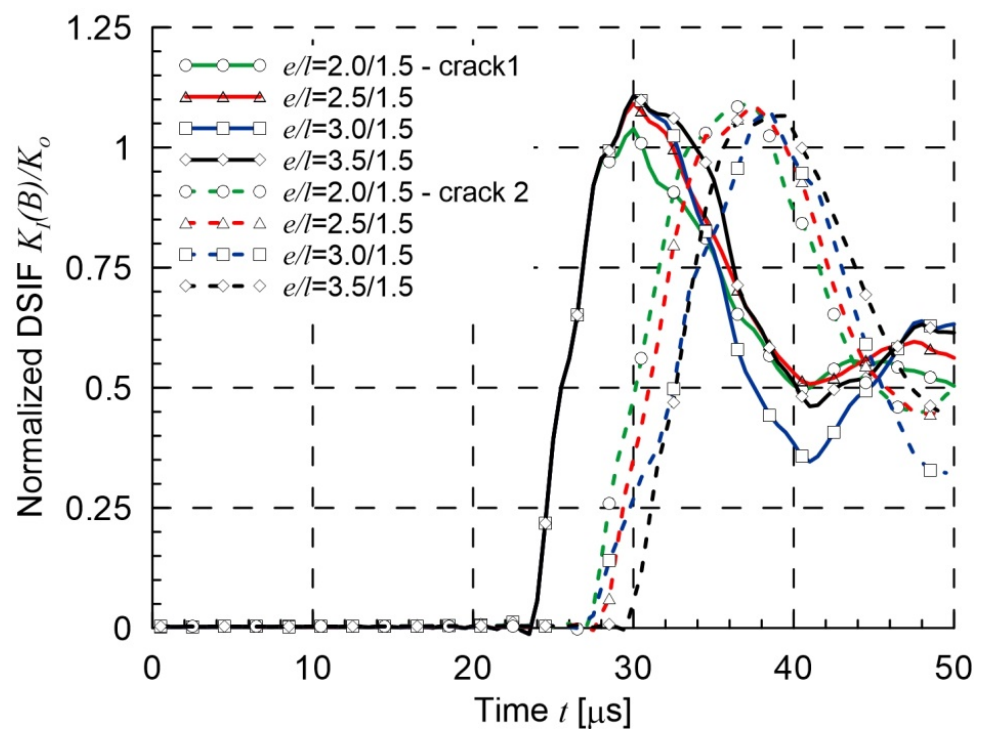

Fig. 9. Normalized DSIF $K_{I} / K_{o}(B)$ for the inclined branches.

\subsection{Different number of branched cracks in an infinite domain}

An influence of different number of interacting cracks shown in Fig. 10 on SIF is studied. The number of cracks varies from one to four. The distance between the cracks is $e=2.0 \mathrm{~cm}$. The normalized static SIF are given in Table 3. When the number of cracks increases the $K_{I} / K_{o}(A)$ increase and $K / K_{o}(B)$ decreases. The normalized dynamic DSIF for four cracks are shown in Fig. 11 and for the first crack tip $A$ and $B$ for different number of cracks in Fig. 12. It is interesting to note in Fig. 11 that $K_{I} / K_{o}(A)$ is gradually increasing for the following cracks. 


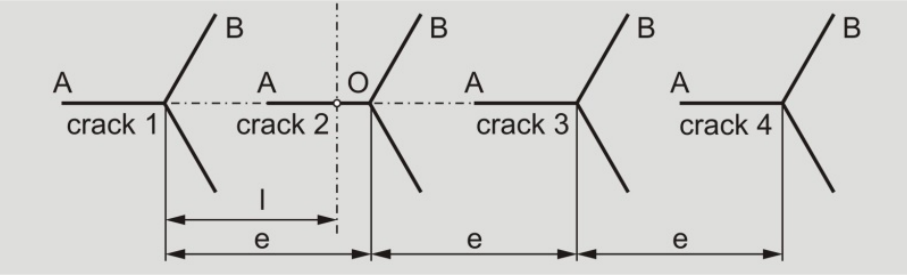

Fig. 10. Different number of branched cracks in an infinite domain.

Table 3. Normalized SIF for different number of branched cracks in an infinite domain.

\begin{tabular}{|c|c|c|c|}
\hline number of cracks & $\boldsymbol{K}_{\boldsymbol{I}}(\boldsymbol{A})$ & $\boldsymbol{K}_{\boldsymbol{I}}(\boldsymbol{B})$ & $\boldsymbol{K}_{\boldsymbol{I I}}(\boldsymbol{B})$ \\
\hline \multicolumn{4}{|c|}{ crack 1 } \\
\hline 1 & 0.016 & 0.754 & 0.541 \\
\hline 2 & 0.032 & 0.614 & 0.488 \\
\hline 3 & 0.041 & 0.600 & 0.472 \\
\hline 4 & 0.047 & 0.595 & 0.461 \\
\hline \multicolumn{4}{|c|}{ crack 2 } \\
\hline 2 & 0.014 & 0.700 & 0.416 \\
\hline 3 & 0.036 & 0.567 & 0.364 \\
\hline 4 & 0.047 & 0.554 & 0.346 \\
\hline \multicolumn{4}{|c|}{ crack 3 } \\
\hline 3 & 0.053 & 0.689 & 0.392 \\
\hline 4 & 0.076 & 0.558 & 0.340 \\
\hline \multicolumn{4}{|c|}{ crack 4 } \\
\hline 4 & 0.069 & 0.684 & 0.381 \\
\hline
\end{tabular}

\section{Conclusions}

Multiple branched cracks in an infinite plate subjected to static and dynamic loadings were considered. The influence of orientation, distance and number of cracks on stress intensity factors was studied. Static SIF for branches parallel to the applied loading are small but the dynamic DSIF have significant values. The dynamic opening DSIF for inclined branches with respect to the loading are larger than DSIF for the parallel branches. The dynamic opening DSIF for multiple cracks are the smallest for the parallel branch of the first crack and the largest for the last crack.

\section{Acknowledgement}

The scientific research is financed by National Science Centre, Poland, in years 2016-2019, grant no. 2015/19/B/ST8/02629. 


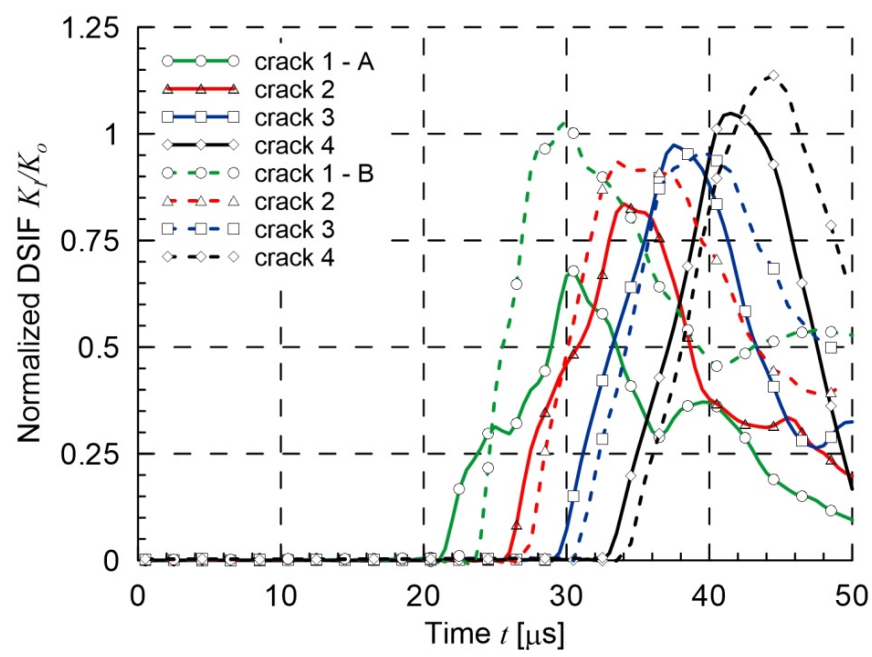

Fig. 11. Normalized DSIF $K_{I} / K_{o}$ for four branched cracks in an infinite domain.

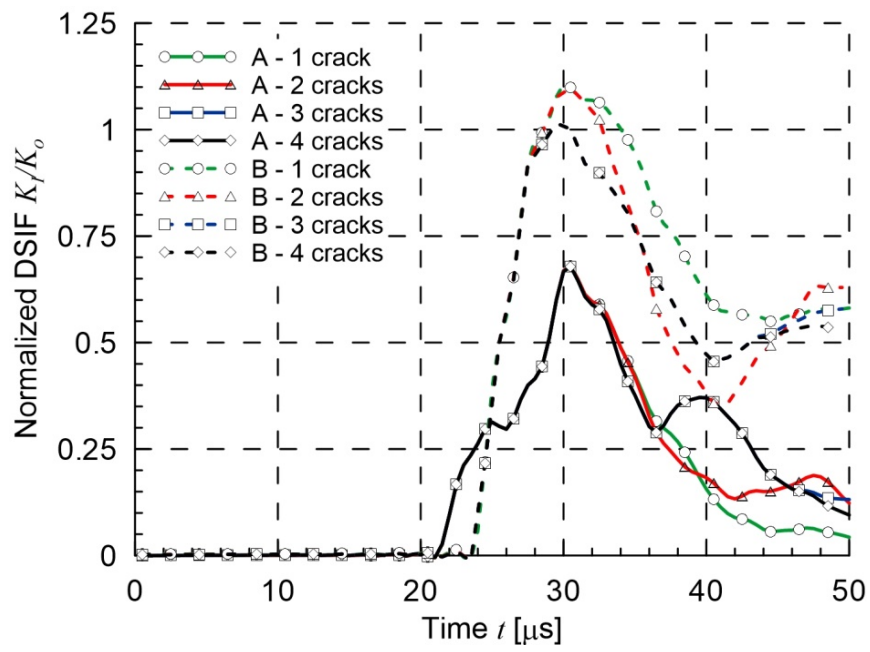

Fig. 12. Normalized DSIF $K_{I} / K_{o}$ for the first crack for different number of branched cracks in an infinite domain.

\section{References}

1. Ch. Daux, N. Moes, J. Dolbow, N. Sukumar, T. Belytschko, Int. J. Numer. Meth. Engng, 48, 1741-1760 (2000)

2. S .Raffie, Th. Seelig, D. Gross. Int. J. Frac., 120, 545-561 (2003).

3. A. Portela, M. H. Aliabadi, D. P. Rooke, Int. J. Numer. Meth. Engng, 33, 1269-1287 (1992)

4. P. Fedeliński, M. H. Aliabadi, D.P. Rooke, Comp. Struct., 59, 1021-1031 (1996)

5. P. Fedeliński, Engng Anal. Bound. Elem., 28, 1135-1147 (2004)

6. P. Fedeliński P., J. Civil Engng, Envir. Arch., 34, 17-26 (2017) 